\title{
Value of the New 5-Tiered Prostate Cancer Grade Group System on Predicting Oncological Outcomes for Radical Prostatectomy Population in Korea
}

\author{
Minhyun Cho', Sangjun Yoo ${ }^{2}$, Juhyun Park ${ }^{2}$, Chang Wook Jeong ${ }^{1}$, Ja Hyeon $\mathrm{Ku}^{1}$, \\ Cheol Kwak', Hyeon Hoe Kim ${ }^{1}$, Min Chul Cho ${ }^{2}$, Hyeon Jeong ${ }^{2}$ \\ ${ }^{1}$ Department of Urology, Seoul National University Hospital, Seoul, Korea \\ ${ }^{2}$ Department of Urology, Seoul Metropolitan Government - Seoul National University Boramae Medical Center, Seoul, Korea
}

\begin{abstract}
Purpose: We evaluated the prognostic value of the 5-tiered grade group in Korean patients who underwent radical prostatectomy.

Materials and Methods: Between 1996 and 2016, a number of 2,883 consecutive patients who underwent radical prostatectomy were included for the analysis. The impacts of biopsy and pathologic grade group on predicting biochemical recurrence $(\mathrm{BCR})$ were assessed using multivariate analysis. Median follow-up duration was 49.0 months.

Results: Mean age was 66.5 years and prostate-specific antigen (PSA) was $11.8 \mathrm{ng} / \mathrm{mL}$. Prostate cancer was locally advanced on magnetic resonance imaging in 13.4\%. Biopsy grade group was as follows: 1 (46.8\%), 2 $(19.8 \%), 3(14.2 \%), 4(14.1 \%)$, and $5(5.1 \%)$. Pathology stage was $\leq \mathrm{T} 2$ in $63.6 \%$, T3a in $26.0 \%$, and T3b/T4 in $10.4 \%$ patients. Pathologic grade was as follows: $1(31.3 \%), 2(37.9 \%), 3(20.2 \%), 4(4.7 \%)$, and $5(5.1 \%)$. In multivariate analysis using biopsy-related variables, biopsy grade group (1, reference; 2 , hazard ratio [HR], 1.771; $p=0.001 ; 3, H R, 2.736 ; p<0.001 ; 4, H R, 2.966 ; p<0.001 ; 5, H R, 3.707 ; p<0.001$ ) was associated with BCR-free survival, PSA level and \% positive core. In multivariate analysis using pathologic outcomes, pathologic grade group (1, reference; 2, HR, 1.882; $p<0.001 ; 3$, HR, 3.352; $p<0.001$; 4, HR, 3.890; $p<0.001 ; 5$, HR: 3.118, $p<0.001$ ) was associated with BCR-free survival in addition to pathologic stage and positive surgical margin.

Conclusions: New 5-tiered grading system could be useful for predicting oncological outcomes in Korean patients although its role for distinguishing outcomes between patients with grade groups 3-5 need to be validated before wide application of this grade system in Korea. (Korean J Urol Oncol 2018;16:75-81)
\end{abstract}

Key Words: Neoplasm grading $\cdot$ Prostate neoplasms $\cdot$ Prostatectomy $\cdot$ Recurrence

Received July 17, 2018, Revised August 10, 2018,

Accepted August 13, 2018

Corresponding Author: Hyeon Jeong

Department of Urology, Seoul Metropolitan Government - Seoul National University Boramae Medical Center, 20 Boramae-ro 5-gil, Dongjak-gu, Seoul 07061, Korea

E-mail: drjeongh @ gmail.com

Tel: +82-2-870-2177, Fax: +82-2-870-3863

ORCID code: https://orcid.org/0000-0002-0744-1402

\section{INTRODUCTION}

The incidence of prostate cancer in Korea has dramatically increased in recent years. ${ }^{1}$ This increase is associated with an aging population, westernization of dietary habits, and widespread use of prostate-specific antigen (PSA) testing. ${ }^{2}$ In 1999, prostate cancer was the 10th most common cancer in Korean men, but became the 5 th most common cancer by $2014{ }^{1}$

D'Amico's classification has been the most widely accepted risk stratification model for predicting the prognosis of prostate

(i) (1) This is an Open Access article distributed under the terms of the Creative Commons Attribution Non-Commercial License (http://creativecommons.org/licenses/by-nc/4.0/) which permits unrestricted non-commercial use, distribution, and reproduction in any medium, provided the original work is properly cited. 2018 (C) Copyright The Korean Urological Oncology Society and The Korean Prostate Society. All Rights Reserved. 
cancer. $^{3-5}$ This system divides prostate cancer into low-, intermediate-, and high-risk disease using the Gleason score, serum PSA level, and Clinical T stage. ${ }^{6}$ According to D'Amico's classification, a Gleason score of 6 is regarded as low risk, 7 as intermediate risk, and $\geq 8$ as high risk. ${ }^{6}$ However, as treatment methods for prostate cancer are varied and individualized, more accurate classification of prostate cancer grade is clinically needed. In this regard, in 2014, a new grading system for prostate cancer was introduced by the International Society of Urological Pathology (ISUP), which divided the Gleason score into 5 groups. ${ }^{7-10}$ After the introduction of the new 5-tiered grading system, several Western studies validated the predictive role of the new system and reported its superiority over the previous grading systems. ${ }^{7,9,10}$

However, the new 5-tiered grading system has not been validated in Asian prostate cancer patients. Because prostate cancer in Asians is reported to have more aggressive pathological characteristics $^{11}$ and worse oncological outcomes than that in Western patients, ${ }^{12-15}$ the prognostic value of the new system in Asian prostate cancer patients needs to be validated before its clinical application. Therefore, we aimed to evaluate the prognostic value of the new 5-tiered grading system, with respect to both biopsy and pathologic grade, in Korean patients with prostate cancer who underwent radical prostatectomy.

\section{MATERIALS AND METHODS}

The present study protocol was reviewed and approved by the Seoul National University Hospital Institutional Review Board (approval number: 16-2014-145). Informed consent was exempted by the board. Medical records of consecutive patients who underwent radical prostatectomy for prostate cancer from 1996 to 2016 at Seoul National University Hospital or Boramae Medical Center were retrospectively reviewed. Patients with suspected metastasis on preoperative imaging studies, patients with positive lymph node on pathologic examination, and/or patients who underwent adjuvant or neoadjuvant treatment were excluded from the analysis. Finally, 2,883 patients, including 2,273 patients from Seoul National University Hospital and 610 patients from Boramae Medical Center, who underwent radical prostatectomy for prostate cancer were included in the analysis.

In all patients with biopsy-proven prostate cancer, preoperative prostate magnetic resonance imaging (MRI) was performed at our institute. However, $21.5 \%$ of patients who under- went MRI in an external hospital or whose MRI reading did not use the proper format had no studies available for analysis. MRI findings were divided into 2 groups: locally confined and locally advanced. Bone scanning was also performed in patients with biopsy-proven prostate cancer. Operative methods were selected by clinicians after sufficient patient counseling regarding tumor characteristics and patient's preference. During surgery, lymph node dissection was performed in patients with intermediate- or high-risk prostate cancer and/or patients with suspicious lymph node enlargement observed in preoperative imaging studies. Specimens obtained through prostate biopsy or surgery were interpreted by pathologists with urologic expertise. Tumor grade was divided into 5 groups according to the new grading system proposed by the ISUP. ${ }^{7}$ Group 1 was replaced with a Gleason score of 6 , group 2 with a Gleason score of $7(3+4)$, group 3 with a Gleason score of $7(4+3)$, group 4 with a Gleason score of 8 , and group 5 with a Gleason score of 9 or 10 . The primary outcome of this study is biochemical recurrence (BCR) after surgery, which was defined as postoperative serum PSA level exceeding $0.2 \mathrm{ng} / \mathrm{mL}$ in 2 consecutive tests. ${ }^{16,17}$ After surgery, patients visited the outpatient clinic and followed up with PSA level testing, according to the National Comprehensive Cancer Network guidelines. ${ }^{18}$ The median follow-up duration was 49.0 months.

Among patient and tumor characteristics, categorical variables were expressed by using a frequency table and continuous variables were expressed as mean \pm standard deviation (SD). Clinical $\mathrm{T}$ stage, based on the findings of digital rectal examination, was categorized into 3 groups (clinical T1c/T2a, $\mathrm{T} 2 \mathrm{~b} / \mathrm{T} 2 \mathrm{c}$, and T3-4). The $\%$ positive core on prostate biopsy was calculated as follows: positive core on prostate biopsy/total biopsy core $\times 100$. Pathologic stage was categorized into 4 groups (T2a/T2b vs. T2c vs. T3a vs. T3b/4). Kaplan-Meier analysis with log rank test was performed to compare BCR-free survival based on the biopsy and pathologic grade group. To assess the predictive value of biopsy grade group on BCR-free survival, univariate and multivariate Cox regression analysis was performed using preoperative variables. The predictive value of pathologic grade group on BCR-free survival was also assessed using univariate and multivariate analysis after adjusting for patient characteristics and pathology-related variables.

Among patient and tumor characteristics, categorical variables were expressed by using a frequency table, and continuous variables were expressed as mean \pm SD. Kaplan-Meier analysis 
with log rank test was performed to compare BCR-free survival based on the biopsy and pathologic grade group. To assess the predictive value of biopsy grade group on BCR-free survival, univariate and multivariate Cox regression analysis was performed using preoperative variables. The impacts of preoperative variables and surgical pathology related variables on BCR were assessed using univariate and multivariable analysis, respectively. Variables with $\mathrm{p}<0.2$ on univariate analysis were selected for the multivariable analysis. All statistical analyses were performed using IBM SPSS Statistics ver. 21.0 (IBM Co., Armonk, NY, USA). A p-value of less than 0.05 was considered statistically significant.

\section{RESULTS}

Mean age at surgery was 66.5 years and body mass index was $24.2 \mathrm{~kg} / \mathrm{m}^{2}$ (Table 1). Hypertension and diabetes were present in $1,326(46.0 \%)$ and 444 patients (15.4\%), respectively. Mean preoperative serum PSA level was $11.8 \mathrm{ng} / \mathrm{mL}$ and prostate volume was $40.5 \mathrm{~mL}$. Mean \% positive core was $31.8 \%$. Biopsy grade group was as follows: 1,349 patients (46.8\%) in group 1, $571(19.8 \%)$ in group 2, $410(14.2 \%)$ in group 3, 406 $(14.1 \%)$ in group 4 , and $147(5.1 \%)$ in group 5. Preoperative clinical stage was T1c/T2a, T2b/T2c, and T3-4 in 2,541 (88.1\%), $254(8.8 \%)$, and 88 patients $(3.1 \%)$, respectively. Disease was locally confined in $1,878(65.1 \%)$ and locally advanced in 386 patients (13.4\%) as observed through MRI. Pathologic stage was T2 or less in 1,833 patients (63.6\%), T3a in $750(26.0 \%)$, and T3b/T4 in 300 patients (10.4\%). Pathologic grade was 1 in 902 patients (31.3\%), 2 in 1,094 (37.9\%), 3 in 583 (20.2\%), 4 in 136 (4.7\%), and 5 in 147 (5.1\%). Surgical margin was positive for prostate cancer in 997 patients (34.6\%).

In Kaplan-Meier analysis, the 5-year BCR-free survival was $87.0 \%, 73.8 \%, 71.3 \%, 58.4 \%$, and $44.4 \%$ in grade groups $1-5$, respectively (Fig. 1). BCR-free survival rates were significantly different according to biopsy grade group, except for groups 2 and 3. The 5-year BCR-free survival for pathologic grade group was as follows: $89.0 \%$ in group $1,78.6 \%$ in group 2, $63.0 \%$ in group 3, $54.8 \%$ in group 4 , and $47.8 \%$ in group 5 . BCR-free survival was not significantly different between grade groups 3,4 , and 5 although it was significantly different between grade groups 1,2 , and 3 .

In univariate analysis, age, PSA level, clinical stage, prostate volume, \% positive core, MRI, and biopsy Gleason grade group
Table 1. Baseline characteristics $(n=2,883)$

\begin{tabular}{|c|c|}
\hline Variable & Value \\
\hline Age (yr) & $66.5 \pm 6.6$ \\
\hline BMI $\left(\mathrm{kg} / \mathrm{m}^{2}\right)$ & $24.2 \pm 2.7$ \\
\hline Hypertension & $1326(46.0)$ \\
\hline Diabetes & $444(15.4)$ \\
\hline PSA $(n g / m L)$ & $11.8 \pm 14.5$ \\
\hline Prostate volume $(\mathrm{mL})$ & $40.5 \pm 18.0$ \\
\hline$\%$ Positive core $(\%)$ & $31.8 \pm 22.4$ \\
\hline \multicolumn{2}{|l|}{ Biopsy Grade group } \\
\hline 1 & $1,349(46.8)$ \\
\hline 2 & $571(19.8)$ \\
\hline 3 & $410(14.2)$ \\
\hline 4 & $406(14.1)$ \\
\hline 5 & $147(5.1)$ \\
\hline \multicolumn{2}{|l|}{ Clinical stage } \\
\hline $\mathrm{T} 1 \mathrm{c} / \mathrm{T} 2 \mathrm{a}$ & $2,541(88.1)$ \\
\hline $\mathrm{T} 2 \mathrm{~b} / \mathrm{T} 2 \mathrm{c}$ & $254(8.8)$ \\
\hline $\mathrm{T} 3 / \mathrm{T} 4$ & $88(3.1)$ \\
\hline \multicolumn{2}{|l|}{ MRI findings } \\
\hline Not available & $619(21.5)$ \\
\hline Locally confined & $1878(65.1)$ \\
\hline Locally advanced & $386(13.4)$ \\
\hline \multicolumn{2}{|l|}{ Pathologic Grade group } \\
\hline 1 & $902(31.3)$ \\
\hline 2 & $1,094(37.9)$ \\
\hline 3 & $583(20.2)$ \\
\hline 4 & $136(4.7)$ \\
\hline 5 & $168(5.8)$ \\
\hline \multicolumn{2}{|l|}{ Pathology stage } \\
\hline $\mathrm{T} 2$ or less & $1833(63.6)$ \\
\hline $\mathrm{T} 3 \mathrm{a}$ & $750(26.0)$ \\
\hline $\mathrm{T} 3 \mathrm{~b} / \mathrm{T} 4$ & $300(10.4)$ \\
\hline Positive surgical margin & $997(34.6)$ \\
\hline
\end{tabular}

Values are presented as mean \pm standard deviation or number (\%). BMI: body mass index, PSA: prostate-specific antigen, MRI: magnetic resonance imaging.

were significantly associated with BCR-free survival (Table 2). In multivariate analysis with preoperative variables, biopsy grade group (group 1, reference; group 2, hazard ratio [HR], 1.771; $\mathrm{p}=0.001$; group 3, HR, 2.736; $\mathrm{p}<0.001$; group 4, HR, $2.966 ; \mathrm{p}<0.001$; group 5 , HR, 3.707; $\mathrm{p}<0.001$ ) was significantly associated with BCR-free survival, in addition to preoperative serum PSA level (HR, 1.010; $\mathrm{p}=0.018)$ and \% positive core (HR, 1.007; $\mathrm{p}=0.005)$. In univariate analysis with pathology-related variables, pathologic stage, pathologic Gleason group, and the presence of surgical margin were significantly associated with BCR-free survival, in addition to preoperative variables (Table 3). In multivariate analysis, patho- 

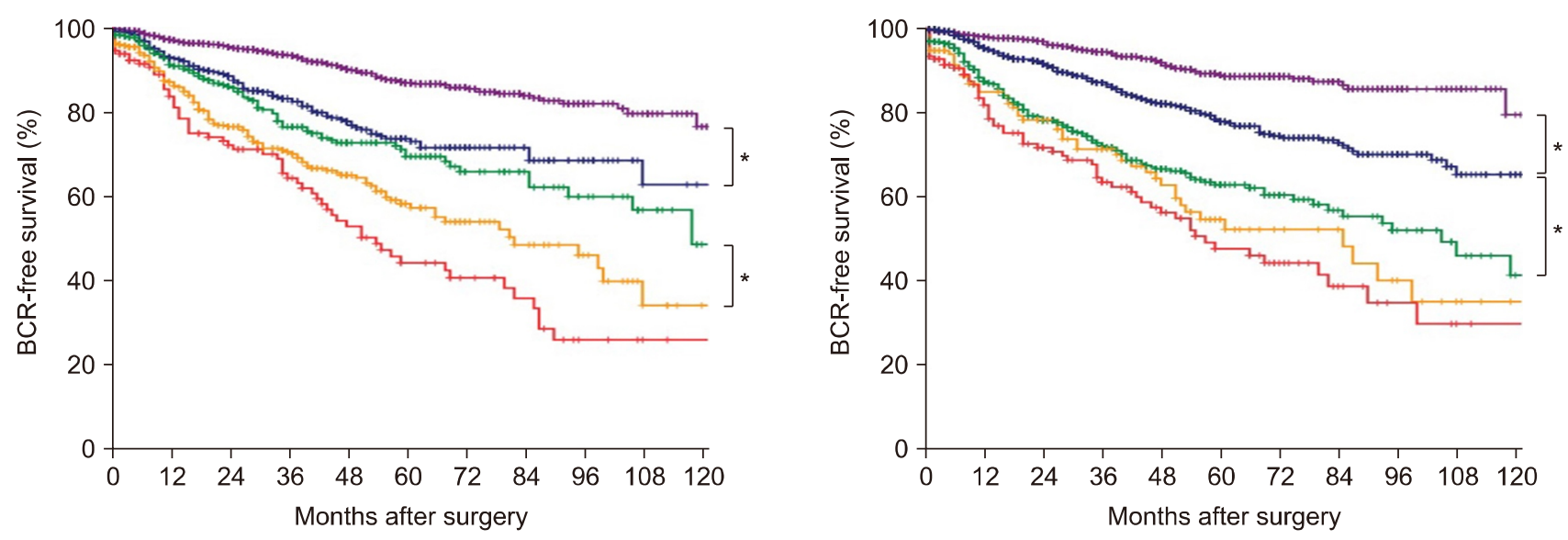

Fig. 1. (A) Biochemical recurrence (BCR)-free survival according to biopsy grade group. (B) BCR-free survival according to pathologic grade group. Grade 1, purple; grade 2, blue; grade 3, green; grade 4, orange; grade 5 , red. ${ }^{*} \mathrm{p}<0.05$.

Table 2. Multivariate analysis for predicting biochemical recurrence: preoperative variables

\begin{tabular}{|c|c|c|c|c|}
\hline \multirow{2}{*}{ Variable } & \multicolumn{2}{|c|}{ Univariate } & \multicolumn{2}{|c|}{ Multivariate } \\
\hline & HR $(95 \% \quad C I)$ & p-value & HR $(95 \% \mathrm{CI})$ & p-value \\
\hline Age (continuous) & $1.016(1.002-1.029)$ & 0.023 & $0.998(0.978-1.018)$ & 0.843 \\
\hline BMI (continuous) & $1.005(0.974-1.038)$ & 0.738 & & \\
\hline Hypertension (yes vs. no) & $1.060(0.890-1.262)$ & 0.512 & & \\
\hline Diabetes (yes vs. no) & $1.058(0.831-1.346)$ & 0.648 & & \\
\hline PSA level (continuous) & $1.016(1.013-1.019)$ & $<0.001$ & $1.010(1.002-1.019)$ & 0.018 \\
\hline \multicolumn{5}{|l|}{ Clinical stage } \\
\hline $\mathrm{T} 1 \mathrm{c} / \mathrm{T} 2 \mathrm{a}$ & Reference & & Reference & \\
\hline $\mathrm{T} 2 \mathrm{~b} / \mathrm{T} 2 \mathrm{c}$ & $1.505(1.112-2.036)$ & 0.008 & $0.702(0.301-1.633)$ & 0.411 \\
\hline $\mathrm{T} 3 / \mathrm{T} 4$ & $3.664(2.542-5.281)$ & $<0.001$ & $1.526(0.545-4.272)$ & 0.421 \\
\hline Prostate volume & $0.993(0.988-0.998)$ & 0.012 & $0.998(0.989-1.006)$ & 0.575 \\
\hline$\%$ Positive core (continuous) & $1.019(1.015-1.023)$ & $<0.001$ & $1.007(1.002-1.019)$ & 0.005 \\
\hline \multicolumn{5}{|l|}{ Biopsy Gleason group } \\
\hline 1 & Reference & & Reference & \\
\hline 2 & $2.239(1.729-2.900)$ & $<0.001$ & $1.771(1.252-2.505)$ & 0.001 \\
\hline 3 & $2.814(2.146-3.690)$ & $<0.001$ & $2.736(1.959-3.822)$ & $<0.001$ \\
\hline 4 & $4.352(3.405-5.563)$ & $<0.001$ & $2.966(2.099-4.192)$ & $<0.001$ \\
\hline 5 & $6.039(4.479-8.144)$ & $<0.001$ & $3.707(2.428-5.659)$ & $<0.001$ \\
\hline MRI (locally advanced vs. confined) & $1.775(1.394-2.259)$ & $<0.001$ & $1.153(0.775-1.715)$ & 0.484 \\
\hline
\end{tabular}

HR: hazard ratio, CI: confidence interval, BMI: body mass index, PSA: prostate-specific antigen, MRI: magnetic resonance imaging.

logic grade group (group 1, reference; group 2, HR, 1.882; $\mathrm{p}$ $<0.001$; group 3, HR, 3.352; $<<0.001$; group 4, HR, 3.890; $\mathrm{p}<0.001$; group 5, HR, 3.118; $\mathrm{p}<0.001$ ) was significantly associated with BCR-free survival, in addition to pathologic stage (T2a or less, reference; T3a, HR, 1.590; $\mathrm{p}<0.001$; T3b or greater, HR, 1.830; $\mathrm{p}=0.001)$ and positive surgical margin (HR, $1.736, \mathrm{p}<0.001)$.

\section{DISCUSSION}

The new 5-tiered grading system has proven its value in predicting oncological outcomes in Western patients with prostate cancer. ${ }^{7,19}$ However, its value in Asian prostate cancer patients remained to be determined before it could be clinically applied. Considering the worse pathological characteristics and oncological outcomes of the disease in Asians than in Western pa- 
Table 3. Multivariate analysis for predicting biochemical recurrence: surgical pathology related variables

\begin{tabular}{|c|c|c|c|c|}
\hline \multirow{2}{*}{ Variable } & \multicolumn{2}{|c|}{ Univariate } & \multicolumn{2}{|c|}{ Multivariate } \\
\hline & $\mathrm{HR}(95 \% \mathrm{CI})$ & p-value & $\mathrm{HR}(95 \% \mathrm{CI})$ & p-value \\
\hline Age (continuous) & $1.016(1.002-1.029)$ & 0.023 & $0.987(0.968-1.005)$ & 0.0154 \\
\hline BMI (continuous) & $1.005(0.974-1.038)$ & 0.738 & & \\
\hline Hypertension (yes vs. no) & $1.060(0.890-1.262)$ & 0.512 & & \\
\hline Diabetes (yes vs. no) & $1.058(0.831-1.346)$ & 0.648 & & \\
\hline PSA level (continuous) & $1.016(1.013-1.019)$ & $<0.001$ & $1.009(0.998-1.019)$ & 0.098 \\
\hline \multicolumn{5}{|l|}{ Clinical stage } \\
\hline $\mathrm{T} 1 \mathrm{c} / \mathrm{T} 2 \mathrm{a}$ & Reference & & Reference & \\
\hline $\mathrm{T} 2 \mathrm{~b} / \mathrm{T} 2 \mathrm{c}$ & $1.505(1.112-2.036)$ & 0.008 & $0.712(0.323-1.570)$ & 0.400 \\
\hline $\mathrm{T} 3 / \mathrm{T} 4$ & $3.664(2.542-5.281)$ & $<0.001$ & $1.255(0.454-3.474)$ & 0.661 \\
\hline Prostate volume (continuous) & $0.993(0.988-0.998)$ & 0.012 & $0.998(0.990-1.006)$ & 0.585 \\
\hline MRI (locally advanced vs. confined) & $1.775(1.394-2.259)$ & $<0.001$ & $1.243(0.867-1.782)$ & 0.237 \\
\hline \multicolumn{5}{|l|}{ Pathologic stage } \\
\hline $\mathrm{T} 2$ or less & Reference & & Reference & \\
\hline $\mathrm{T} 3 \mathrm{a}$ & $2.250(1.846-2.741)$ & $<0.001$ & $1.590(1.241-2.037)$ & $<0.001$ \\
\hline $\mathrm{T} 3 \mathrm{~b}$ or greater & $4.287(3.407-5.394)$ & $<0.001$ & $1.830(1.299-2.576)$ & 0.001 \\
\hline \multicolumn{5}{|l|}{ Pathologic Gleason group } \\
\hline 1 & Reference & & Reference & \\
\hline 2 & $2.245(1.706-2.954)$ & $<0.001$ & $1.882(1.369-2.586)$ & $<0.001$ \\
\hline 3 & $4.661(3.528-6.158)$ & $<0.001$ & $3.352(2.397-4.688)$ & $<0.001$ \\
\hline 4 & $5.756(3.989-8.307)$ & $<0.001$ & $3.890(2.403-6.296)$ & $<0.001$ \\
\hline 5 & $7.038(5.030-9.848)$ & $<0.001$ & $3.118(1.971-4.930)$ & $<0.001$ \\
\hline Positive surgical margin (yes vs. no) & $2.620(2.201-3.119)$ & $<0.001$ & $1.736(1.385-2.177)$ & $<0.001$ \\
\hline
\end{tabular}

HR: hazard ratio, CI: confidence interval, BMI: body mass index, PSA: prostate-specific antigen, MRI: magnetic resonance imaging.

tients, ${ }^{11,13-15}$ it was not possible to apply the new grading system as is, on Asians. In the current study, in more than one-third of the patients, the disease was locally advanced (as observed through on pathologic examination), which was significantly higher than the results of Western studies, even though the PSA level was not higher than that in previous studies. ${ }^{12,20}$ In addition, the Gleason grade was also higher than in some Western studies. In this study, $30.8 \%$ of patients had grade 3-5 disease, which was higher than the $24.1 \%$ reported by a previous Western study, ${ }^{20}$ but was in accordance with the results of another study. ${ }^{13}$ These findings might affect the predictive value of the 5-tiered system. Therefore, its prognostic value needs to be validated in Korean patients.

In this study, the biopsy grade group sequentially predicted the 5-year BCR-free survival, although statistical significance was not achieved between the groups. In other words, the new grading system might be helpful for predicting 5-year BCR-free survival using preoperative and biopsy-related variables. ${ }^{19}$ In addition, preoperative PSA level and \% positive core, well-known predictors of pathologic and oncological out- comes, ${ }^{21}$ were also reported to be significant predictors of BCR-free survival after surgery. Although not a predictor of oncological outcomes, the role of multiparametric MRI needs to be assessed in a future study, because some of the MRI performed in this study was not multiparametric, and a standardized reporting system was not applied. ${ }^{22,23}$ In previous studies, MRI was reportedly valuable for predicting tumor characteristics before surgery. ${ }^{24}$

However, in pathologic grade, the new 5-tiered system may have only limited value in predicting prognosis of prostate cancer after radical prostatectomy, especially in patients with grade groups 3-5. This study found no difference between pathologic grade groups $3-5$, which was different from the finding in Western studies. ${ }^{20}$ These results might be due to the small number of patients and/or the relatively long period of enrollment of patients. Because the oncological outcome of prostate cancer in Korea has considerably improved in recent years, ${ }^{25}$ long study periods could explain these findings. According to the National Statistical Office, the 5-year survival rate for prostate cancer was only $55.9 \%$ between 1993 and 1995, but was $67.2 \%$ 
between 1996 and 2000, 80.3\% between 2001 and 2005, and 91.1\% between 2006 and 2010. In the period between 2011 and 2014 , the survival rate rose sharply to $93.3 \%$. $^{1}$ These improvements in prostate cancer survival are thought to be mostly the result of improved oncological outcomes in patients with high-risk prostate cancer, ${ }^{26}$ which might influence the results of the current study. However, based on this study, grade groups 2 and 3, previously called Gleason score $7(3+4)$ and Gleason score $7(4+3)$, respectively, showed significantly different BCR-free survival, which was in accordance with previous studies. ${ }^{27,28}$ In this regard, patients with Gleason score 7 need to be further divided into 2 groups, and treatment/prognosis needs to be explained based on these results. Because grades 2 and 3 account for more than half of all patients, the value of the new system should not be overlooked, although its value in patients with grade $\geq 3$ needs to be carefully interpreted.

Moreover, another limitation of the current study is that biopsy and pathologic Gleason score have not been reviewed by pathologists using 2005 ISUP Gleason system. In this regards, the changes in Gleason score over time cannot be adjusted in the current study. ${ }^{29}$ Although some argued that the impacts of Gleason system change on patient management and prognosis is uncertain, ${ }^{30}$ more studies with a pathologic review of the Gleason score thought to be needed to validate the results of the current study.

Final limitation of the current study thought to be selection bias. Because we excluded a considerable proportion of patients with aggressive pathologic features before analysis, the results of the current need to be interpreted with cautions.

The current study has several limitations, including the retrospective design. Although this was one of the largest Korean studies in this field, an even larger number of patients needs to be included for the analysis to accurately evaluate the role of the grading system for prostate cancer, especially in Gleason grade groups $\geq 4$. In addition, the relatively long enrollment period is another limitation which might affect the results of the current study. Although this study may only be applicable to nonmetastatic prostate cancer patients who underwent radical prostatectomy, the results may be of value to clinicians, because this large-scale Asian study assessed the predictive value of the new 5-tiered grading system.

\section{CONCLUSIONS}

The new 5-tiered grading system could be useful for predicting oncological outcomes in Korean patients with prostate cancer with Gleason grade groups 1-3. However, its role for distinguishing outcomes between patients with Gleason grade groups $3-5$ of the disease needs to be validated before application of this system in Korea.

\section{CONFLICT OF INTEREST}

The authors claim no conflicts of interest.

\section{REFERENCES}

1. Korean Statistical Information Service [Internet]. Daejeon (Korea): Statistics Korea; [cited 2017 Jun 15]. Available from: http://kosis.kr.

2. Han HH, Park JW, Na JC, Chung BH, Kim CS, Ko WJ. Epidemiology of prostate cancer in South Korea. Prostate Int 2015;3:99-102.

3. Ouzzane A, Koenig P, Ballereau C, Zini L, Ghoneim T, Maladry F, et al. Oncologic outcomes after radical prostatectomy: French validation of the D'Amico risk group classification. Prog Urol 2010;20:1206-12.

4. Boorjian SA, Karnes RJ, Rangel LJ, Bergstralh EJ, Blute ML. Mayo Clinic validation of the D'amico risk group classification for predicting survival following radical prostatectomy. J Urol 2008;179:1354-60.

5. D'Amico AV, Whittington R, Malkowicz SB, Schultz D, Blank K, Broderick GA, et al. Biochemical outcome after radical prostatectomy, external beam radiation therapy, or interstitial radiation therapy for clinically localized prostate cancer. JAMA 1998;280:969-74.

6. Hernandez DJ, Nielsen ME, Han M, Partin AW. Contemporary evaluation of the D'amico risk classification of prostate cancer. Urology 2007;70:931-5.

7. Epstein JI, Egevad L, Amin MB, Delahunt B, Srigley JR, Humphrey PA, et al. The 2014 International Society of Urological Pathology (ISUP) Consensus Conference on Gleason Grading of Prostatic Carcinoma: Definition of Grading Patterns and Proposal for a New Grading System. Am J Surg Pathol 2016;40:244-52.

8. Gordetsky J, Epstein J. Grading of prostatic adenocarcinoma: current state and prognostic implications. Diagn Pathol 2016;11:25.

9. Epstein JI, Zelefsky MJ, Sjoberg DD, Nelson JB, Egevad L, Magi-Galluzzi $\mathrm{C}$, et al. A Contemporary Prostate Cancer 
Grading System: a validated alternative to the Gleason score. Eur Urol 2016;69:428-35.

10. Kryvenko ON, Epstein JI. Changes in prostate cancer grading: Including a new patient-centric grading system. Prostate 2016;76:427-33.

11. Jeong IG, Dajani D, Verghese M, Hwang J, Cho YM, Hong $\mathrm{JH}$, et al. Differences in the aggressiveness of prostate cancer among Korean, Caucasian, and African American men: A retrospective cohort study of radical prostatectomy. Urol Oncol 2016;34:3.e9-14.

12. Kang DI, Chung JI, Ha HK, Min K, Yoon J, Kim W, et al. Korean prostate cancer patients have worse disease characteristics than their American counterparts. Asian Pac J Cancer Prev 2013;14:6913-7.

13. Byun SS, Lee S, Lee SE, Lee E, Seo SI, Lee HM, et al. Recent changes in the clinicopathologic features of Korean men with prostate cancer: a comparison with Western populations. Yonsei Med J 2012;53:543-9.

14. Lin SS, Clarke CA, Prehn AW, Glaser SL, West DW, O'Malley CD. Survival differences among Asian subpopulations in the United States after prostate, colorectal, breast, and cervical carcinomas. Cancer 2002;94:1175-82.

15. Robbins AS, Koppie TM, Gomez SL, Parikh-Patel A, Mills PK. Differences in prognostic factors and survival among white and Asian men with prostate cancer, California, 1995-2004. Cancer 2007;110:1255-63.

16. Freedland SJ, Humphreys EB, Mangold LA, Eisenberger M, Dorey FJ, Walsh PC, et al. Risk of prostate cancer-specific mortality following biochemical recurrence after radical prostatectomy. JAMA 2005;294:433-9.

17. Cookson MS, Aus G, Burnett AL, Canby-Hagino ED, D'Amico AV, Dmochowski RR, et al. Variation in the definition of biochemical recurrence in patients treated for localized prostate cancer: the American Urological Association Prostate Guidelines for Localized Prostate Cancer Update Panel report and recommendations for a standard in the reporting of surgical outcomes. J Urol 2007;177:540-5.

18. Chung MS, Lee SH, Lee DH, Kim SJ, Kim CS, Lee KS, et al. Practice patterns of Korean urologists for screening and managing prostate cancer according to PSA level. Yonsei Med J 2012;53:1136-41.

19. Giunchi F, Brunocilla E, Borghesi M, Rizzi S, Ricci MS, Romagnoli D, et al. Revised Gleason grading system is a better predictor of indolent prostate cancer at the time of diagnosis: retrospective clinical-pathological study on matched biopsy and radical prostatectomy specimens. Clin Genitourin Cancer 2014;12:325-9.

20. Spratt DE, Cole AI, Palapattu GS, Weizer AZ, Jackson WC,
Montgomery JS, et al. Independent surgical validation of the new prostate cancer grade-grouping system. BJU Int 2016;118:763-9.

21. D'Amico AV, Whittington R, Malkowicz SB, Schultz D, Fondurulia J, Chen MH, et al. Clinical utility of the percentage of positive prostate biopsies in defining biochemical outcome after radical prostatectomy for patients with clinically localized prostate cancer. J Clin Oncol 2000;18:1164-72.

22. Tanimoto A, Nakashima J, Kohno H, Shinmoto H, Kuribayashi S. Prostate cancer screening: the clinical value of diffusion-weighted imaging and dynamic MR imaging in combination with T2-weighted imaging. J Magn Reson Imaging 2007;25:146-52.

23. Weinreb JC, Barentsz JO, Choyke PL, Cornud F, Haider MA, Macura KJ, et al. PI-RADS Prostate Imaging - Reporting and Data System: 2015, Version 2. Eur Urol 2016;69:16-40.

24. Jeong CW, Ku JH, Moon KC, Hong SK, Byun SS, Cho JY, et al. Can conventional magnetic resonance imaging, prostate needle biopsy, or their combination predict the laterality of clinically localized prostate cancer? Urology 2012;79:1322-7.

25. Lee DH, Jung HB, Chung MS, Lee SH, Chung BH. The change of prostate cancer treatment in Korea: 5 year analysis of a single institution. Yonsei Med J 2013;54:87-91.

26. Yu J, Kwon YS, Kim S, Han CS, Farber N, Kim J, et al. Pathological outcome following radical prostatectomy in men with prostate specific antigen greater than $10 \mathrm{ng} / \mathrm{ml}$ and histologically favorable risk prostate cancer. J Urol 2016;195: 1464-70.

27. Waliszewski P, Wagenlehner F, Kribus S, Schafhauser W, Weidner W, Gattenlöhner S. Objective grading of prostate carcinoma based on fractal dimensions: Gleason $3+4=7 \mathrm{a}$ $\neq$ Gleason $4+3=7 \mathrm{~b}$. Urologe A 2014;53:1504-11.

28. Koontz BF, Tsivian M, Mouraviev V, Sun L, Vujaskovic Z, Moul J, et al. Impact of primary Gleason grade on risk stratification for Gleason score 7 prostate cancers. Int J Radiat Oncol Biol Phys 2012;82:200-3.

29. Montironi R, Cheng L, Lopez-Beltran A, Scarpelli M, Mazzucchelli R, Mikuz G, et al. Original Gleason system versus 2005 ISUP modified Gleason system: the importance of indicating which system is used in the patient's pathology and clinical reports. Eur Urol 2010;58:369-73.

30. Van Praet C, Libbrecht L, D'Hondt F, Decaestecker K, Fonteyne V, Verschuere S, et al. Agreement of Gleason score on prostate biopsy and radical prostatectomy specimen: is there improvement with increased number of biopsy cylinders and the 2005 revised Gleason scoring? Clin Genitourin Cancer 2014;12:160-6. 